\title{
Extracellular DNA-induced antimicrobial peptide resistance in Salmonella enterica serovar Typhimurium
}

\author{
Lori Johnson ${ }^{1 \dagger}$, Shawn R Horsman ${ }^{1 \dagger}$, Laetitia Charron-Mazenod ${ }^{1}$, Amy L Turnbull', Heidi Mulcahy ${ }^{1}$,
} Michael G Surette ${ }^{1,2}$ and Shawn Lewenza ${ }^{*}$

\begin{abstract}
Background: The Salmonella enterica serovar Typhimurium PhoPQ two component system (TCS) is activated by low $\mathrm{Mg}^{2+}$ levels, low pH and by antimicrobial peptides (AP). Under $\mathrm{Mg}^{2+}$ limitation, the PhoPQ system induces pmrD expression, which post-translationally activates the PmrAB TCS. PhoPQ and PmrAB control many genes required for intracellular survival and pathogenesis. These include the polymyxin resistance (pmr) operon, which is required for aminoarabinose modification of LPS and protecting the outer membrane from antimicrobial peptide disruption and killing. Extracellular DNA is a ubiquitous polymer in the matrix of biofilms and accumulates in some infection sites. Extracellular DNA chelates cations and thus activates the Pseudomonas aeruginosa PhoPQ/PmrAB systems, leading to expression of the orthologous arn (pmr) operon.
\end{abstract}

Results: Here we show that extracellular DNA induces expression of the $S$. Typhimurium pmr antimicrobial peptide resistance operon in a PhoPQ and PmrAB-dependent manner. Induction of the pmr genes by DNA was blocked when present with excess $\mathrm{Mg}^{2+}$. Exogenous DNA led to increased resistance of planktonic cultures to aminoglycosides, antimicrobial peptides (AP) and ciprofloxacin, but only AP resistance was PhoPQ/PmrAB-dependent. Extracellular DNA was shown to be a matrix component of $S$. Typhimurium biofilms cultivated in flow chambers and on glass surfaces. A pmrH-gfp fusion was highly expressed in flow chamber biofilms cultivated in medium with repressing levels of $10 \mathrm{mM} \mathrm{Mg}{ }^{2+}$ and co-localized with eDNA. Expression of $\mathrm{pmrH}$-lux was monitored in plastic peg biofilms and shown to require PhoPQ and PmrAB. Biofilms had higher levels of pmrH expression compared to planktonic cultures. We propose that DNA accumulation in biofilms contributes to the increased pmrH-lux expression in biofilms.

Conclusions: The Salmonella PhoPQ/PmrAB systems and antimicrobial peptide resistance are activated by the cation chelating properties of extracellular DNA. DNA-induced AP resistance may allow immune evasion and increased survival of S. Typhimurium biofilms formed during extracellular growth stages of an infection or outside the host.

Keywords: Salmonella, phoPQ, pmrAB, Antimicrobial peptides, Antibiotic resistance, Extracellular DNA, Biofilms, Immune evasion

\footnotetext{
* Correspondence: slewenza@ucalgary.ca

†Equal contributors

'Department of Microbiology, Immunology and Infectious Diseases, University of Calgary, HRIC Building, Room 2C66, 3330 Hospital Drive NW, Calgary, AB T2N 4N1, Canada

Full list of author information is available at the end of the article
}

\section{Biomed Central}

(c) 2013 Johnson et al.; licensee BioMed Central Ltd. This is an Open Access article distributed under the terms of the Creative Commons Attribution License (http://creativecommons.org/licenses/by/2.0), which permits unrestricted use, distribution, and reproduction in any medium, provided the original work is properly cited. 


\section{Background}

Salmonella enterica serovar Typhimurium is an enteroinvasive bacterial pathogen typically encountered by ingesting contaminated food or water. S. Typhimurium causes self-limiting gastroenteritis in humans and typhoidlike fever in mice [1,2]. Greater than $99 \%$ of the bacteria in murine salmonellosis are killed in the stomach or passed out of the gut [2], but $S$. Typhimurium that survive passage through the acidic stomach environment enter into the small intestine, where upon they transverse the intestinal epithelial barrier. The bacteria are then phagocytosed by macrophages or they can actively invade both phagocytic and non-phagocytic cells using a type III secretion system [1]. Following invasion, Salmonella disseminates throughout the body leading to a systemic typhoid-like infection [2].

Salmonella forms biofilms on abiotic surfaces such as plastic and egg conveyer belts, which may have a role in environmental survival of this organism [3,4]. Biofilm formation and aggregation in S. enterica serovar Typhimurium is exemplified by the rdar colony morphology, where colonies grown on media containing Congo red are red, dry, and rough [5,6]. This morphology requires the production of curli fimbriae and multiple exopolysaccharides [7,8]. $S$. Typhimurium also grows enmeshed in EPS rich biofilms on the surface of gallstones, which may contribute to inefficient antibiotic treatment and facilitates typhoid carriage $[9,10]$. Biofilm shedding from colonized gallstones is likely a source of recurring infections [11].

The PhoPQ two-component system is important for intracellular survival within macrophages. Limiting $\mathrm{Mg}^{2+}$, low $\mathrm{pH}$ and the presence of antimicrobial peptides are PhoPQ-activating signals in culture $[12,13]$ but low $\mathrm{pH}$ and antimicrobial peptides are important activating signals during intracellular macrophage growth [14]. The PmrAB two component system responds to $\mathrm{Fe}^{3+}$ and low $\mathrm{pH}$, and is activated under $\mathrm{Mg}^{2+}$ limiting conditions by a posttranslational mechanism involving PmrD, a PhoPQregulated protein. PmrD prevents the dephosphorylation of PmrA by PmrB, thus activating the expression of PmrAregulated genes [15]. The pmrHFIJKLM operon is directly regulated by $\mathrm{PmrAB}$, is induced during phagocytosis and is required for survival from host antimicrobial peptide production [16]. The pmr operon encodes an LPS modification system that is responsible for aminoarabinose modification of the lipid A moiety of LPS. Reducing the negative charge of the bacterial surface with aminoarabinose is critical for reducing the membrane damaging effects of cationic antimicrobial peptides.

We recently demonstrated that DNA is a cation chelator that induces expression of the Pseudomonas aeruginosa arnBCADTEF-ugd (PA3552-PA3559; pmr) operon in DNA-enriched planktonic cultures and biofilms [17]. DNA sequesters cations and creates a condition that resembles a
$\mathrm{Mg}^{2+}$-limited environment, similar to known chelators like EDTA. Expression of this operon was required for very high levels of biofilm resistance to antimicrobial peptides and partially contributed to aminoglycoside resistance [17]. During $\mathrm{Mg}^{2+}$ limiting growth conditions, the $P$. aeruginosa PhoPQ and PmrAB systems are both required for expression of the arn operon [18,19]. Both the PhoPQ and $\mathrm{PmrAB}$ systems respond to $\mathrm{Mg}^{2+}$ limitation in $P$. aeruginosa, and there is no PmrD ortholog to connect the two pathways. In addition, the $P$. aeruginosa PhoQ sensor does not directly detect antimicrobial peptides, and the PmrB sensor does not respond to trivalent metals [18]. Extracellular DNA also induces the expression of PmrABregulated spermidine synthesis genes, which results in the production of the polycation spermidine on the surface and protection of the outer membrane from antimicrobial peptide treatment [20]. Both the arn and spermidine synthesis (PA4773-PA4775) clusters were induced in biofilms formed by a $b f m R$ mutant of $P$. aeruginosa that accumulated more eDNA than wild-type biofilms [21]. When sufficient DNA accumulates in $P$. aeruginosa biofilms, or in the cystic fibrosis (CF) lung where the concentration of DNA is very high and leads to viscous sputum production in CF patients $[22,23]$, the expression of these DNA-induced surface modifications likely protect from host antimicrobial peptide killing. Therefore, we wanted to determine if extracellular DNA plays a general role in antimicrobial peptide resistance by imposing a cation limitation on $S$. Typhimurium biofilms and activating the $\mathrm{PhoPQ} / \mathrm{PmrAB}$ systems, similar to $P$. aeruginosa.

\section{Results and discussion}

\section{Extracellular DNA induces expression of the Salmonella} pmr operon

A low copy, plasmid-encoded transcriptional lux fusion to the pmrH promoter [24] was expressed in Salmonella enterica serovar Typhimurium 14208 under various planktonic growth conditions. At $\mathrm{pH} 7.4$, the $\mathrm{pmrH}$-lux reporter was repressed at $1 \mathrm{mM} \mathrm{Mg}{ }^{2+}$ but was induced 13-fold in a stepwise fashion as the $\mathrm{Mg}^{2+}$ concentration was decreased to $0.06 \mathrm{mM}$ (Figure 1A). The pmrH-lux fusion was most highly expressed under low $\mathrm{pH}$ (5.5) (Figure 1B), even with the addition of up to $50 \mathrm{mM} \mathrm{Mg}{ }^{2+}$ (data not shown). These observations were consistent with previous reports of $p m r$ expression conditions [12,13]. A fixed concentration of DNA $(0.5 \%, 5 \mathrm{mg} / \mathrm{ml})$ was added to cultures grown in a range of $\mathrm{Mg}^{2+}$ concentrations between $1 \mathrm{mM}$ and $0.06 \mathrm{mM}$. In each $\mathrm{Mg}^{2+}$ concentration, the addition of $0.5 \%$ DNA caused a strong induction of pmrH-lux expression (up to 70-fold) (Figure 1C). To confirm that DNA induced pmrH-lux expression via cation chelation, we added exogenous $5 \mathrm{mM} \mathrm{Mg}{ }^{2+}$, which was sufficient to prevent DNA-mediated induction of pmrH-lux (Figure 1C). Taken 

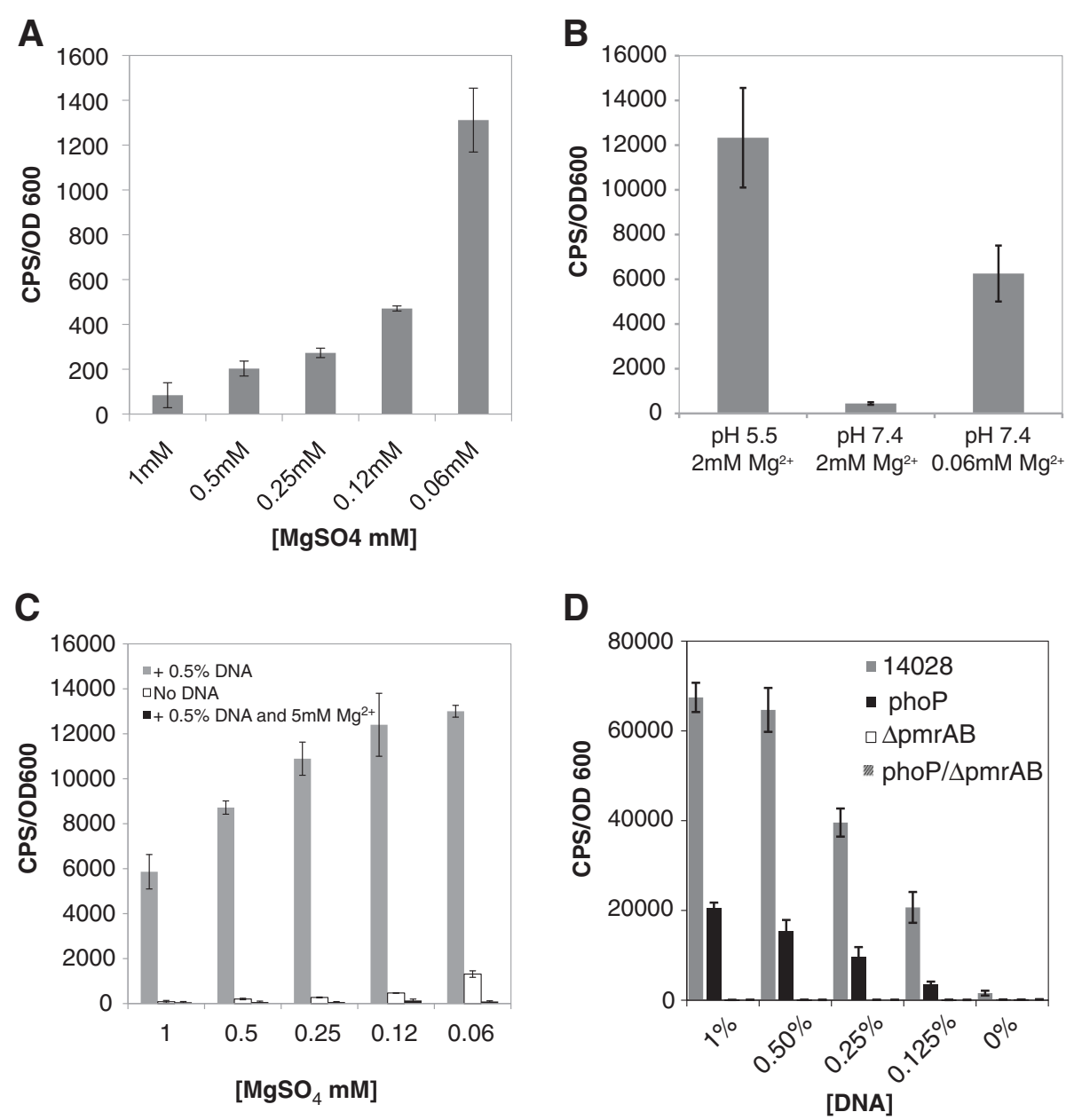

Figure 1 Cation chelation by extracellular DNA induces expression of the pmr operon. (A) Expression of pmrH-lux in $\mathrm{NM} 2 \mathrm{media}$ ( $\mathrm{pH} 7.4$ ) in final $\mathrm{Mg}^{2+}$ concentrations ranging from $1 \mathrm{mM}$ to $0.06 \mathrm{mM}$. (B) Expression of pmrH-lux in NM2 media pH5.5 and pH7.4, in varying $\mathrm{Mg}^{2+}$ concentrations. (C) Expression of pmrH-lux in NM2 media ( $\mathrm{pH7.4)}$ in varying $\mathrm{Mg}^{2+}$ concentrations ranging from $1 \mathrm{mM}$ to $0.06 \mathrm{mM}$ (white bars), media supplemented with $0.5 \%$ DNA ( $5 \mathrm{mg} / \mathrm{ml}$ ) (grey bars) or $0.5 \%$ DNA plus excess $5 \mathrm{mM} \mathrm{Mg}^{2+}$ (black bars). (D) Expression of pmrH-lux in NM2 media (pH7.4) containing repressing levels of $\mathrm{Mg}^{2+}(1 \mathrm{mM})$ and supplemented with increasing concentrations of extracellular DNA, as indicated. Expression was measured in strains 14028, phoP, pmrAB and phoP/pmrAB mutants. In all experiments, gene expression was measured every 20 minutes for 18 hours and the maximal gene expression $(t=\sim 7$ hrs $)$ is shown. The values shown are the means from experiments done in triplicate and the error bars represent the standard deviation.

together, these observations indicate that DNA chelates and sequesters $\mathrm{Mg}^{2+}$ and the cation chelating activity can be blocked with excess $\mathrm{Mg}^{2+}$.

Next, we monitored pmrH-lux expression in wild type, phoPQ, $\triangle p m r A B$ and $p h o P Q / \triangle p m r A B$ mutant backgrounds. DNA-induced expression did not occur in $\triangle p m r A B$ or $p h o P Q / \triangle p m r A B$ double mutants, indicating an absolute requirement for pmrAB in responding to extracellular DNA (Figure 1D). A phoPQ mutant was still able to partially respond to extracellular DNA, which was likely due to the presence of PmrAB (Figure 1D). In summary, extracellular DNA imposes a cation limitation on $S$. Typhimurium, leading to induction of the $\mathrm{pmrH}$ promoter in a PhoP and PmrA-dependent manner.
Extracellular DNA is a matrix component $S$. Typhimurium biofilms

While radar colony biofilms and biofilms on gallstones produce an extracellular matrix composed of multiple EPS species, the presence of extracellular DNA has not been well reported $[5,6]$. Here we cultivated flow chamber biofilms of $S$. enterica serovar Typhimurium at $37^{\circ} \mathrm{C}$ for 48 hours. To determine if DNA accumulates in the matrix of $S$. Typhimurium biofilms, we stained for the presence of extracellular DNA with Toto-1. Large aggregates formed within 2 days that were $20-30 \mu \mathrm{M}$ in height and stained positive for extracellular DNA (Figure 2A-C), illustrating that eDNA accumulates in Salmonella flow chamber biofilms. Biofilms were also cultivated on glass cover slips 
immersed in growth media and stained with propidium iodide, which showed the accumulation of eDNA fibers extending from an aggregate (Figure 2D,E).

\section{DNA-enriched planktonic cultures show increased antibiotic resistance}

The presence of extracellular DNA may lead to increased $S$. Typhimurium pmr expression, increased AP resistance and thus help to explain the antibiotic resistance phenotype that is characteristic of biofilms. To determine the influence of DNA on antibiotic resistance, we tested the antibiotic susceptibility of $S$. Typhimurium 14028 planktonic cultures in the presence and absence of exogenous DNA (pH 7.4). The addition of $0.5 \%$ DNA $(5 \mathrm{mg} / \mathrm{ml})$ led to a 16-fold increased resistance to polymyxin B and colistin, a 4-fold increased resistance to gentamicin and a $>4$ fold increase in resistance to ciprofloxacin (Table 1). Both phoPQ and pmrAB mutants did not demonstrate DNAinduced resistance to polymyxin $B$ and colistin. However, both mutants had parental levels of DNA-induced resistance to gentamicin and ciprofloxacin, indicating that resistance to these antibiotics was independent of the phoPQ and pmrAB systems (Table 1). Extracellular DNA is known to bind to aminoglycosides through electrostatic interactions [25], and it was recently shown that exogenous DNA shields $P$. aeruginosa from aminoglycoside killing, independent of the $p m r$ resistance mechanism [26].

The observation that phoPQ and $p m r A B$ mutants showed an increased susceptibility to colistin and polymyxin $B$, in the presence of eDNA, indicated a role for PhoPQ/PmrABregulated phenotypes in resistance to membrane acting antimicrobial peptides, likely through the aminoarabinose modification of LPS via the $p m r$ operon.
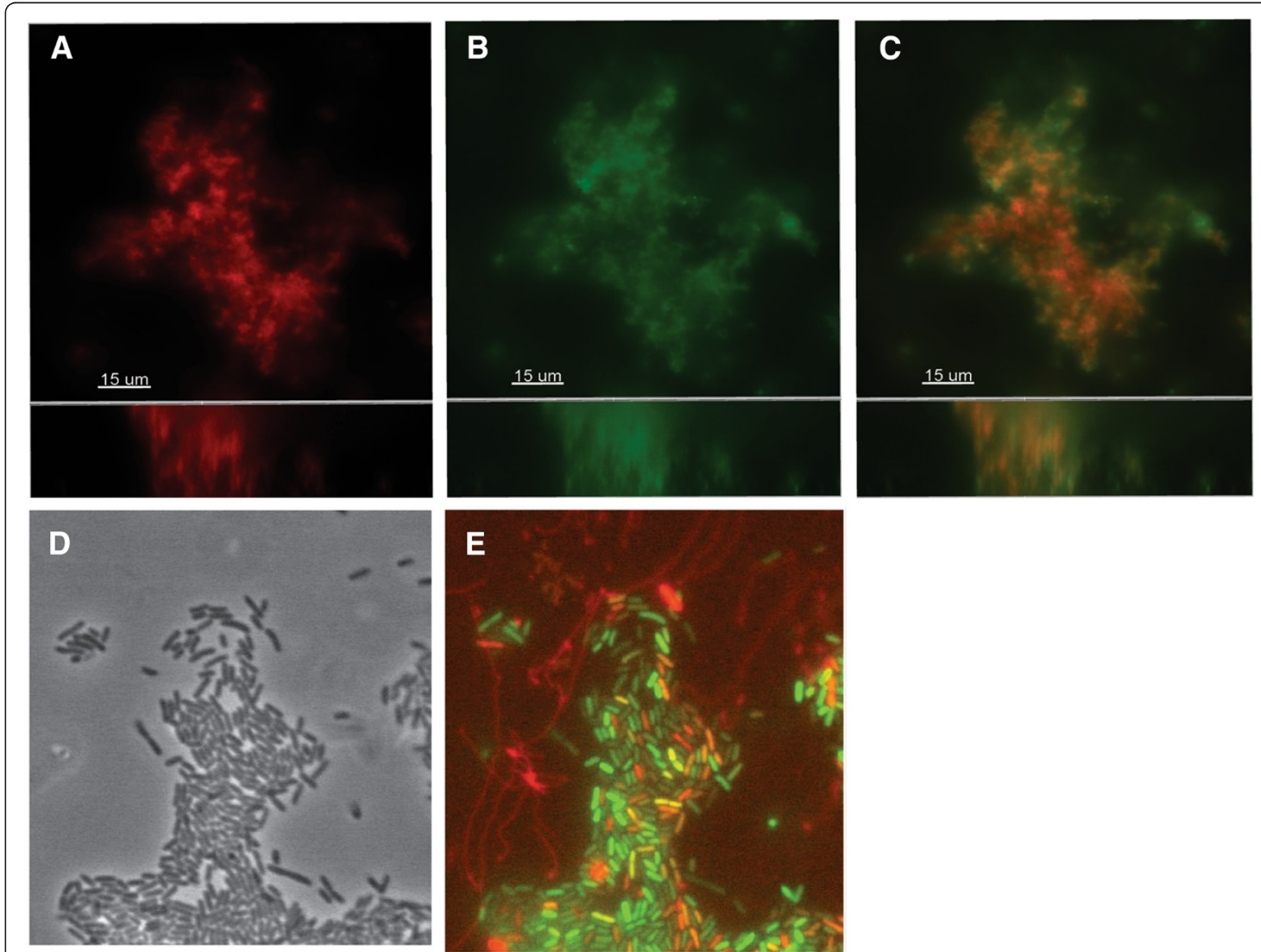

Figure 2 Extracellular DNA accumulates in the matrix of S. Typhimurium biofilms. Biofilms of strain 14028 were cultivated in flow chambers at $37^{\circ} \mathrm{C}$ for 2 days in LB medium and stained for extracellular DNA. Cells in the biofilm were stained with the membrane staining dye FM 4-64 (A). The middle panel depicts the accumulation of extracellular DNA with TOTO-1 staining (B). The images are merged on the right (C). The large image shows the xy plane and the bottom panel shows the xz plane. The scale bar equals $15 \mu \mathrm{M}$. The wild-type 14028 strain carrying the pmrH-gfp construct forms aggregates on the surface of glass $(\mathbf{D})$. The merged image of green fluorescence from pmr expression and red from propidium iodide staining, which stains both dead cells and extracellular DNA (E). 
Table 1 Extracellular DNA induces antibiotic resistance in S. Typhimurium

\begin{tabular}{|c|c|c|c|c|c|c|c|c|}
\hline \multirow[t]{3}{*}{ Strain } & \multicolumn{8}{|c|}{ Minimal inhibitory concentration (MIC) } \\
\hline & \multicolumn{2}{|c|}{ Polymyxin B } & \multicolumn{2}{|c|}{ Colistin } & \multicolumn{2}{|c|}{ Gentamicin } & \multicolumn{2}{|c|}{ Ciprofloxacin } \\
\hline & - & $+\mathrm{DNA}^{\mathrm{a}}$ & - & $+\mathrm{DNA}^{\mathrm{a}}$ & - & $+\mathrm{DNA}^{\mathrm{a}}$ & - & $+\mathrm{DNA}^{\mathrm{a}}$ \\
\hline 14028 & 1 & 16 & 1 & 16 & 0.125 & 0.5 & 0.125 & $>0.5$ \\
\hline phoPQ & 1 & 0.5 & 1 & 1 & 0.125 & 0.25 & 0.125 & $>0.5$ \\
\hline$p m r A B$ & 0.5 & 0.5 & 0.5 & 0.5 & 0.125 & 0.5 & 0.125 & $>0.5$ \\
\hline
\end{tabular}

a The minimal inhibitory concentration (MIC) values were determined in NM2 medium containing $1 \mathrm{mM} \mathrm{Mg}^{2+}(\mathrm{pH} 7.4)$ with or without the addition of $0.5 \%$ fish sperm DNA-sodium salt $(5 \mathrm{mg} / \mathrm{ml})$.

\section{The pmr operon is highly expressed in biofilms}

We wanted to determine if $\mathrm{pmrH}$ is expressed in biofilms due to the natural accumulation of eDNA released from lysed cells. Flow chamber biofilms were cultivated and monitored for the expression of a $p m r H$ - $g f p$ transcriptional fusion. As a positive control, biofilms were cultivated

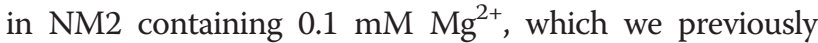
had shown was an inducing condition (Figure 1A). As expected, $p m r H$ - $g f p$ was expressed throughout the biofilm, which also stained positively for extracellular DNA with a second DNA stain Sytox Red, and stained positively for calcofluor white, which binds cellulose and other exopolysaccharides with $\beta-1,4$ linkages (Figure 3 ). We next cultivated biofilms in NM2 containing $0.1 \mathrm{mM} \mathrm{Mg}^{2+}$ for 28 hours and then introduced an extra $10 \mathrm{mM} \mathrm{Mg}^{2+}$ into the media for the next 16 hours of biofilm cultivation. We expected the exogenous addition of $10 \mathrm{mM} \mathrm{Mg}^{2+}$ to repress $\mathrm{pmrH}$ expression since $5 \mathrm{mM} \mathrm{Mg}^{2+}$ could completely repress expression in planktonic cultures in the presence of exogenous DNA (0.5\%). However, pmrH-gfp was strongly expressed in biofilms grown in media despite repressing levels of $\mathrm{Mg}^{2+}$ (Figure 3). Extracellular DNA was visualized in large microcolonies with Sytox Red staining and appeared to generally colocalize with $p m r H-g f p$ expression. This observation suggests that the exogenous addition of excess $\mathrm{Mg}^{2+}$ to pre-formed biofilms could not gain access or was not in sufficient concentration to neutralize the cation chelating properties of endogenous matrix eDNA. Alternatively, the long half-life of Gfp may also contribute to the fluorescence signal detected after 46 hours of growth.

To overcome the potential issue with stable Gfp reporters, we measured gene expression in 96-well format peg-adhered biofilms using the pmrH-lux reporter. In Figure 4A, biofilms cultivated in limiting $\mathrm{Mg}^{2+}(100 \mu \mathrm{M})$ showed the highest expression levels, and expression decreased if biofilms were cultivated in excess $\mathrm{Mg}^{2+}$ conditions $(1-10 \mathrm{mM})$. Biofilms that were cultivated overnight in limiting $\mathrm{Mg}^{2+}$ conditions but were treated with $10 \mathrm{mM} \mathrm{Mg}{ }^{2+}$ for 4 hours, showed a partial repression (Figure 4). This result confirms that the residual pmrH-gfp expression after the addition of excess $\mathrm{Mg}^{2+}$ for 16 hours (Figure 3) is due to stable gfp expression, as the pmrH-lux reporter was responsive to the addition of excess $\mathrm{Mg}^{2+}$.

We measured pmrH-lux expression in conditions with repressing levels of $\mathrm{Mg}^{2+}(1 \mathrm{mM})$, and showed that $\mathrm{pmrH}$ expression was dependent on both PhoPQ and PmrAB in biofilms (Figure 4B). Lastly, we calculated the fold induction values of $p m r H$ between inducing $(100 \mu \mathrm{M})$ and repressing $\mathrm{Mg}^{2+}$ levels $(10 \mathrm{mM})$, simultaneously for both peg-adhered biofilms and the planktonic cultures that served as the inoculum for the biofilms. Interestingly,

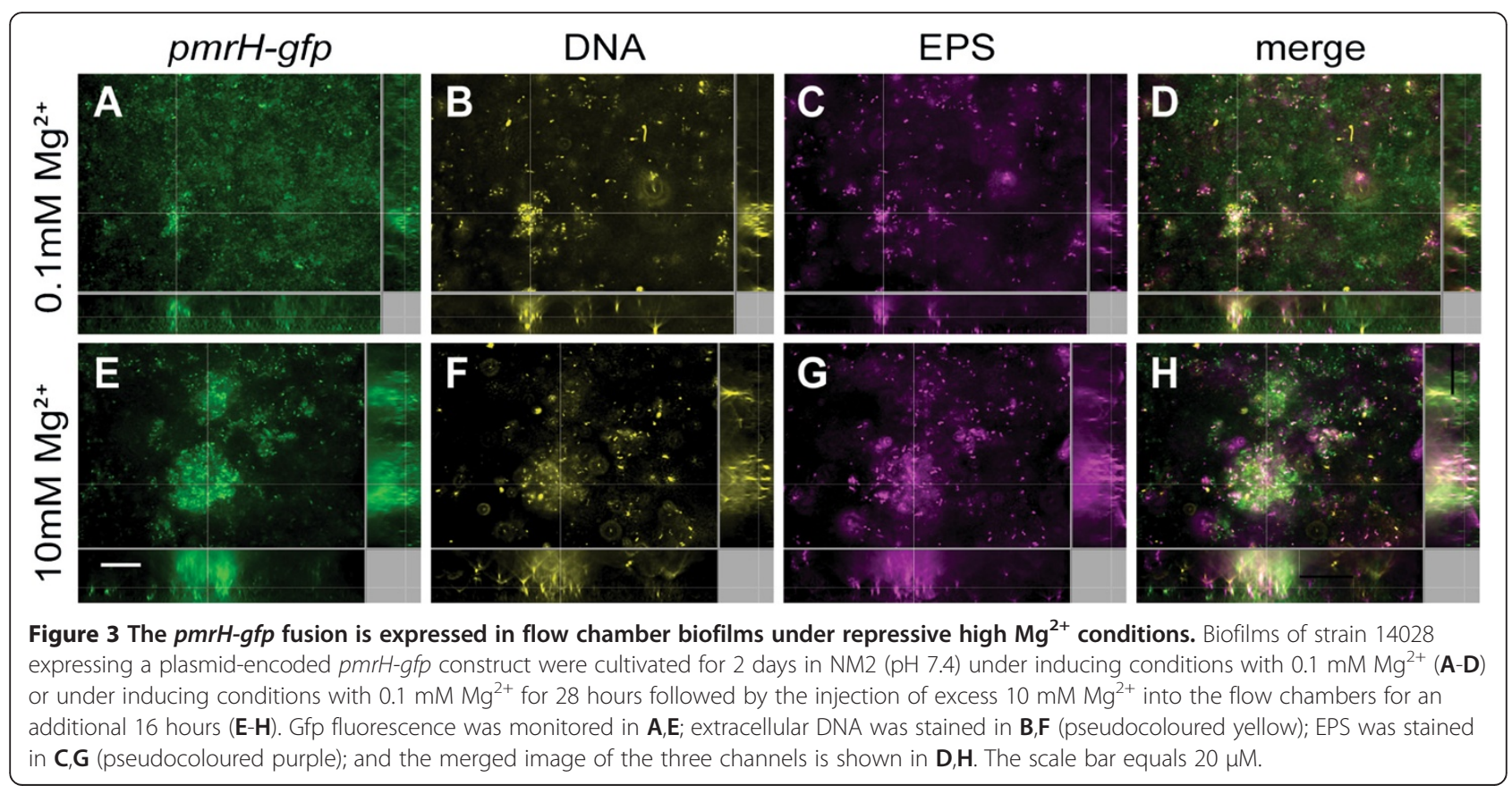




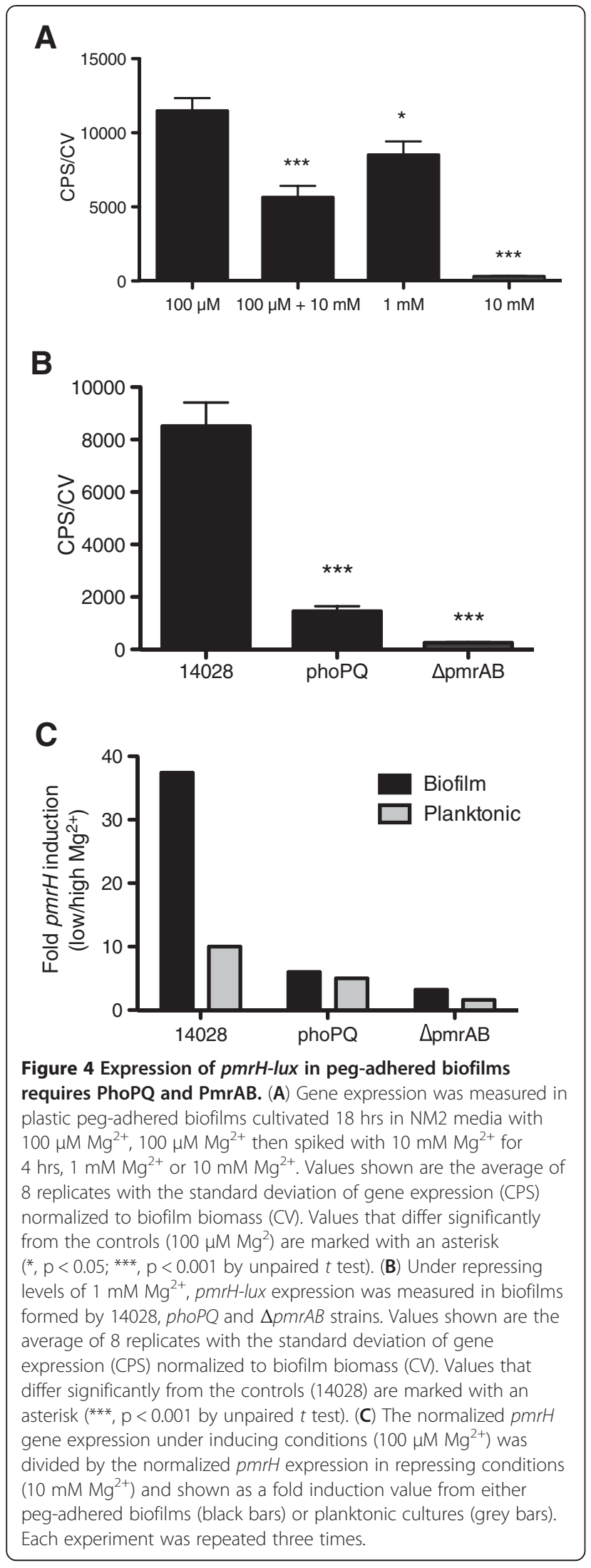

$\mathrm{pmrH}$ was more highly expressed in biofilms when compared to planktonic cultures (27-fold higher), and expression under all conditions required PhoPQ and PmrAB (Figure 4C). We propose that the higher $\mathrm{pmrH}$ expression levels in biofilms may be due to the accumulation of eDNA, which increases $p m r H$ expression in biofilms but not planktonic cultures.

\section{Conclusion}

We showed evidence that extracellular DNA is a component of the $S$. Typhimurium extracellular matrix when grown in biofilms. When added to planktonic cultures, eDNA chelates cations resulting in a $\mathrm{Mg}^{2+}$ limited environment and increased expression of the pmr operon. The $p m r$ operon was more highly expressed in biofilms, when compared to planktonic cultures. Expression of $p m r$ in biofilms and DNA-induced expression in planktonic conditions is dependent on the PhoPQ/PmrAB systems. The addition of eDNA to planktonic cultures also led to increased antimicrobial peptide resistance in a PhoPQ/ PmrAB-dependent manner. Combined with our previous observations of DNA-induced antibiotic resistance mechanisms in $P$. aeruginosa [17], we propose that extracellular DNA has a general role as a cation chelator that induces antimicrobial peptide resistance in biofilms. DNA-induced resistance to antibiotics and antimicrobial peptides from the innate immune system may promote long-term survival of $S$. Typhimurium biofilms in the environment, on the surface of gallstones, or possibly in the extracellular phases of growth during intestinal infection.

\section{Methods}

\section{Bacterial strains and growth conditions}

S. enterica serovar Typhimurium strain ATCC 14028 was used as the reference strain in this study. The phoPQ::Tn10-Tc ${ }^{R}$ mutant was previously described [27], $\triangle p m r A B:: c a t$ was constructed as previously described [28], and the phoPQ $\triangle p m r A B$ mutant strain was constructed by P22-mediated transduction [29] of both mutations into the same background. Cultures were routinely grown overnight at $37^{\circ} \mathrm{C}$ with agitation in Luria Broth base (LB) supplemented with $50 \mu \mathrm{g} / \mathrm{ml}$ kanamycin, if necessary. Gene expression experiments were performed in NM2 defined minimal media with either high (7.4) or low (5.5) $\mathrm{pH}$. NM2 growth medium includes the following components: $5 \mathrm{mM}$ potassium chloride, $7.5 \mathrm{mM}$ ammonium sulfate, $0.5 \mathrm{mM}$ potassium sulfate, $1 \mathrm{mM}$ monopotassium phosphate, $38 \mathrm{mM}$ glycerol, $0.1 \%$ casamino acids, and $100 \mathrm{mM}$ Tris $(\mathrm{pH} 7.4$ or 5.5), supplemented with magnesium sulfate when indicated. When added, the source of extracellular DNA was fish sperm DNA-sodium salt (MJS BioLynx). 


\section{Gene expression assays in planktonic cultures}

Gene expression was performed in high throughput format using 96-well microplates as previously describe [17]. Briefly, overnight cultures were grown in LB supplemented with $50 \mu \mathrm{g} / \mathrm{ml}$ kanamycin as required, diluted 1/1000 into $150 \mu \mathrm{l}$ of NM2 defined culture medium with $\mathrm{MgSO}_{4}$, DNA or both, in 96-well black plates with a transparent bottom (9520 Costar; Corning Inc.) and overlaid with $50 \mu \mathrm{l}$ of mineral oil to prevent evaporation. Microplate planktonic cultures were incubated at $37^{\circ} \mathrm{C}$ in a Wallac Victor ${ }^{3}$ luminescence plate reader (Perkin-Elmer) and optical density (growth, $\mathrm{OD}_{600}$ ) and luminescence (gene expression, counts per second (CPS)) readings were taken every 20 minutes throughout growth.

\section{Biofilm and gene expression assays on pegs}

Biofilms were cultivated on 96-well format, polystyrene pegs (Nunc-TSP) that were immersed in $150 \mu \mathrm{l}$ of NM2 growth medium, as previously described [17]. After biofilm cultivation, non-adherent cells were removed by rinsing the pegs once in $20 \mathrm{mM}$ Tris buffer ( $\mathrm{pH}$ 7.4). Gene expression (CPS) from peg-adhered biofilms was measured by luminescence readings in the Wallac MicroBeta Trilux multi-detector (Perkin-Elmer). Biofilm formation on the pegs was quantitated by crystal violet (CV) staining as previously described [17]. Gene expression (CPS) on pegs was divided by the biofilm biomass (CV) to normalize gene expression to cell number (CPS/ $\mathrm{CV}$ ), and gene expression in planktonic culture was divided by the $\mathrm{OD}_{600}$ value of cells in suspension to normalize for cell number $\left(\mathrm{CPS} / \mathrm{OD}_{600}\right)$. Biofilms were cultivated in NM2 with limiting $\mathrm{Mg}^{2+}(100 \mu \mathrm{M})$ or high levels of $\mathrm{Mg}^{2+}(1-10 \mathrm{mM})$.

\section{Minimal inhibitory concentration (MIC) assay}

The MIC values were determined using the broth microdilution procedure in 96-well microplates. Briefly, all strains were grown overnight in LB medium, sub-cultured into NM2 medium (1 $\mathrm{mM} \mathrm{Mg}^{2+}$ ) (1/100 dilution) and grown to mid-log phase. All cultures were normalized to a common $\mathrm{OD}_{600}$ value and $10 \mu \mathrm{l}$ of mid-log culture $(\sim 6 \times$ $\left.10^{5} \mathrm{cfu}\right)$ was inoculated into $90 \mu \mathrm{l}$ of NM2 media containing repressing levels $\mathrm{Mg}^{2+}(1 \mathrm{mM})$, with or without $5 \mathrm{mg} / \mathrm{ml}$ DNA-sodium salt. Microtitre plates containing the antibiotic dilution series and bacteria were incubated for 18 hours at $37^{\circ} \mathrm{C}$. The MIC was determined as the concentration of antibiotic that reduced growth to an $\mathrm{OD}_{600}$ value less than 0.1. The median MIC values from three experiments are shown.

\section{Flow chamber biofilm cultivation and imaging}

Biofilms were grown in flow chambers with channel dimensions of $1 \times 4 \times 40 \mathrm{~mm}$ as previously described but with minor modifications [30]. Autoclaved silicone tubing (VWR, .062 ID x .125 OD x .032 wall) was assembled and sterilized by pumping $0.5 \%$ hypochlorite solution through the flow chamber for 2 hours. For rinsing, sterile water was pumped though for 30 minutes followed by LB media for 30 minutes. Flow chambers were inoculated by injecting with a syringe, $400 \mu \mathrm{l}$ of mid-log culture diluted to an $\mathrm{OD}_{600}$ of 0.02 . After inoculation, chambers were left without flow for two hours to allow the bacteria to adhere, after which media was pumped though the system at a constant rate of $0.75 \mathrm{rpm}(3.6 \mathrm{ml} /$ hour$)$. Biofilms were cultivated for 48 hours at $37^{\circ} \mathrm{C}$ in $\mathrm{LB}$ medium and stained with the membrane staining dye FM 4-64 (Invitrogen), the extracellular DNA stains TOTO-1 or Sytox Red (Invitrogen), or an EPS stain fluorescent brightener 28 (Sigma). Biofilms were imaged using a Leica DMI $4000 \mathrm{~B}$ widefield fluorescence microscope equipped with filter sets for blue (Ex 390/40, Em 455/50), green (Ex 490/ 20, Em 525/36) and red (Ex 555/25, Em 605/52) fluorescence using the Quorum Angstrom Optigrid (MetaMorph) acquisition software. Images were obtained with a $63 \times 1.4$ objective. Deconvolution was performed with Huygens Essential (Scientific Volume Imaging B.V.) and 3D reconstructions were generated using the Imaris software package (Bitplane AG).

\section{Monitoring pmrH-gfp expression in flow chamber biofilms} The promoter of $p m r H$ was amplified from genomic DNA of S. typhimurium 14028 using the primer pair pmrF-1 (AGTCCTCGAGACTACCGGATGCTGCTTC) and pmr F-2 (AGTCGGATCCATTGCCAGTTAGCCGACA), digested with BamHI-XhoI and cloned into BamHI-XhoI -digested pCS21 upstream of a gfpmut3 reporter [31]. The pmrH-gfp vector was moved into $S$. Typhimurium 14028 by electroporation. Flow chamber biofilms were cultivated in NM2 containing $0.1 \mathrm{mM} \mathrm{Mg}{ }^{2+}$ for 28 hours and then $10 \mathrm{mM} \mathrm{Mg}^{2+}$ was introduced into the growth media for an additional 16 hours of biofilm cultivation prior to imaging.

\section{Authors' contributions}

$\sqcup$ and SRH contributed equally. $L J$ performed gene expression experiments and drafted the manuscript. SRH performed genetic constructions, microscopy experiments and participated in the design of the study. LCM performed gene expression experiments. ALT carried out antimicrobial susceptibility testing. HM and MGS participated in the design of the study. $S L$ conceived of the study, participated in its design, wrote the manuscript and performed experiments. All authors read and approved the final manuscript.

\section{Acknowledgements}

This work is dedicated to the memory of our colleague Dmitry Apel. This research was supported by Cystic Fibrosis Canada, the Canadian Institutes of Health Research the Westaim Corporation, and the Alberta Science and Research Authority (ASRA). We thank Dmitry Apel for strain construction. HM was the recipient of a Cystic Fibrosis Canada fellowship. SL holds the Westaim-ASRA Chair in Biofilm Research. MGS holds a Canada Research Chair in Microbial Gene Expression. 


\section{Author details}

${ }^{1}$ Department of Microbiology, Immunology and Infectious Diseases, University of Calgary, HRIC Building, Room 2C66, 3330 Hospital Drive NW, Calgary, AB T2N 4N1, Canada. ${ }^{2}$ Department of Medicine, Farncombe Family Digestive Health Research Institute, Health Sciences Centre, McMaster University, Rm 3N6-9, 1280 Main Street West, Hamilton, ON L8S 4K1, Canada.

Received: 23 January 2013 Accepted: 17 May 2013

Published: 24 May 2013

\section{References}

1. Ibarra JA, Steele-Mortimer O: Salmonella-the ultimate insider. Salmonella virulence factors that modulate intracellular survival. Cell Microbiol 2009, 11(11):1579-1586.

2. Watson KG, Holden DW: Dynamics of growth and dissemination of Salmonella in vivo. Cell Microbiol 2010, 12(10):1389-1397.

3. Stepanovic S, Cirkovic I, Ranin L, Svabic-Vlahovic M: Biofilm formation by Salmonella spp. and Listeria monocytogenes on plastic surface. Lett Appl Microbiol 2004, 38(5):428-432.

4. Stocki SL, Annett CB, Sibley CD, McLaws M, Checkley SL, Singh N, Surette $M G$, White AP: Persistence of Salmonella on egg conveyor belts is dependent on the belt type but not on the rdar morphotype. Poult Sci 2007, 86(11):2375-2383.

5. Romling U, Bian Z, Hammar M, Sierralta WD, Normark S: Curli fibers are highly conserved between Salmonella typhimurium and Escherichia coli with respect to operon structure and regulation. J Bacterio/ 1998, 180(3):722-731.

6. White AP, Gibson DL, Kim W, Kay WW, Surette MG: Thin aggregative fimbriae and cellulose enhance long-term survival and persistence of Salmonella. J Bacterio/ 2006, 188(9):3219-3227.

7. White AP, Gibson DL, Collinson SK, Banser PA, Kay WW: Extracellular polysaccharides associated with thin aggregative fimbriae of Salmonella enterica serovar enteritidis. J Bacterio/ 2003, 185(18):5398-5407.

8. de Rezende CE, Anriany Y, Carr LE, Joseph SW, Weiner RM: Capsular polysaccharide surrounds smooth and rugose types of Salmonella enterica serovar Typhimurium DT104. Appl Environ Microbiol 2005, 71(11):7345-7351.

9. Prouty AM, Schwesinger WH, Gunn JS: Biofilm formation and interaction with the surfaces of gallstones by Salmonella spp. Infect Immun 2002, 70(5):2640-2649.

10. Crawford RW, Rosales-Reyes R, Ramirez-Aguilar Mde L, Chapa-Azuela O, Alpuche-Aranda C, Gunn JS: Gallstones play a significant role in Salmonella spp. gallbladder colonization and carriage. Proc Natl Acad SCi U S A 2010, 107(9):4353-4358.

11. Gonzalez-Escobedo G, Marshall JM, Gunn JS: Chronic and acute infection of the gall bladder by Salmonella Typhi: understanding the carrier state. Nat Rev Microbiol 2010, 9(1):9-14.

12. Groisman EA: The pleiotropic two-component regulatory system PhoPPhoQ. J Bacteriol 2001, 183(6):1835-1842.

13. Prost $L R$, Miller SI: The Salmonellae PhoQ sensor: mechanisms of detection of phagosome signals. Cell Microbiol 2008, 10(3):576-582.

14. Martin-Orozco N, Touret N, Zaharik ML, Park E, Kopelman R, Miller S, Finlay $B B, G$ ros P, Grinstein S: Visualization of vacuolar acidification-induced transcription of genes of pathogens inside macrophages. Mol Biol Cell 2006, 17(1):498-510.

15. Mitrophanov AY, Groisman EA: Signal integration in bacterial twocomponent regulatory systems. Genes Dev 2008, 22(19):2601-2611.

16. Gunn JS: The Salmonella PmrAB regulon: lipopolysaccharide modifications, antimicrobial peptide resistance and more. Trends Microbiol 2008, 16(6):284-290.

17. Mulcahy H, Charron-Mazenod L, Lewenza S: Extracellular DNA chelates cations and induces antibiotic resistance in Pseudomonas aeruginosa biofilms. PLoS Pathog 2008, 4(11):e1000213.

18. McPhee JB, Lewenza S, Hancock RE: Cationic antimicrobial peptides activate a two-component regulatory system, PmrA-PmrB, that regulates resistance to polymyxin $B$ and cationic antimicrobial peptides in Pseudomonas aeruginosa. Mol Microbiol 2003, 50(1):205-217.

19. McPhee JB, Bains M, Winsor G, Lewenza S, Kwasnicka A, Brazas MD, Brinkman FS, Hancock RE: Contribution of the PhoP-PhoQ and PmrA$\mathrm{PmrB}$ two-component regulatory systems to $\mathrm{Mg} 2+-$ induced gene regulation in Pseudomonas aeruginosa. J Bacteriol 2006, 188(11):3995-4006.

20. Johnson L, Mulcahy H, Kanevets U, Shi Y, Lewenza S: Surface-localized spermidine protects the Pseudomonas aeruginosa outer membrane from antibiotic treatment and oxidative stress. J Bacterio/ 2012, 194(4):813-826.

21. Petrova OE, Schurr JR, Schurr MJ, Sauer K: The novel Pseudomonas aeruginosa two-component regulator BfmR controls bacteriophagemediated lysis and DNA release during biofilm development through PhdA. Mol Microbiol 2011, 81(3):767-783.

22. Ranasinha C, Assoufi B, Shak S, Christiansen D, Fuchs H, Empey D, Geddes D, Hodson M: Efficacy and safety of short-term administration of aerosolised recombinant human DNase I in adults with stable stage cystic fibrosis. Lancet 1993, 342(8865):199-202

23. Shak S, Capon DJ, Hellmiss R, Marsters SA, Baker CL: Recombinant human DNase I reduces the viscosity of cystic fibrosis sputum. Proc Natl Acad Sci U S A 1990, 87(23):9188-9192.

24. Kim W, Surette MG: Swarming populations of Salmonella represent a unique physiological state coupled to multiple mechanisms of antibiotic resistance. Biol Proced Online 2003, 5:189-196.

25. Ramphal R, Lhermitte M, Filliat M, Roussel $P$ : The binding of antipseudomonal antibiotics to macromolecules from cystic fibrosis sputum. J Antimicrob Chemother 1988, 22(4):483-490.

26. Chiang WC, Nilsson M, Jensen PO, Hoiby N, Nielsen TE, Givskov M, TolkerNielsen T: Extracellular DNA shields against aminoglycosides in Pseudomonas aeruginosa Biofilms. Antimicrob Agents Chemother 2013, 57(5):2352-2361

27. Kim W, Killam T, Sood V, Surette MG: Swarm-cell differentiation in Salmonella enterica serovar typhimurium results in elevated resistance to multiple antibiotics. J Bacteriol 2003, 185(10):3111-3117.

28. Nishino K, Hsu FF, Turk J, Cromie MJ, Wosten MM, Groisman EA: Identification of the lipopolysaccharide modifications controlled by the Salmonella PmrA/PmrB system mediating resistance to Fe(III) and $\mathrm{Al}(\mathrm{III})$ Mol Microbiol 2006, 61(3):645-654

29. Maloy SR, Stewart VJ, Taylor RK: Genetic analysis of pathogenic bacteria: A laboratory manual. Plainview, NY: Cold Spring Harbor Laboratory Press; 1996.

30. Horsman SR, Moore RA, Lewenza S: Calcium chelation by alginate activates the type III secretion system in mucoid Pseudomonas aeruginosa biofilms. PLoS One 2012, 7(10):e46826.

31. Bjarnason J, Southward CM, Surette MG: Genomic profiling of ironresponsive genes in Salmonella enterica serovar typhimurium by high-throughput screening of a random promoter library. J Bacteriol 2003, 185(16):4973-4982

doi:10.1186/1471-2180-13-115

Cite this article as: Johnson et al.: Extracellular DNA-induced

antimicrobial peptide resistance in Salmonella enterica serovar Typhimurium. BMC Microbiology 2013 13:115.

\section{Submit your next manuscript to BioMed Central and take full advantage of:}

- Convenient online submission

- Thorough peer review

- No space constraints or color figure charges

- Immediate publication on acceptance

- Inclusion in PubMed, CAS, Scopus and Google Scholar

- Research which is freely available for redistribution 